Artículo de revisión

Rev Urug Cardiol 2018; 33: 220-227

doi: $10.29277 /$ cardio.33.2.6

\title{
Lo mejor del Congreso ACC 2018
}

\author{
Dres. Florencia Cristar', Sebastián Lorenzo², Sofía Noria³
}

\section{Resumen}

Del 10 al 12 de marzo de este año se celebró la reunión número 67 del congreso anual del American College of Cardiology (ACC), la cual tuvo lugar en el Orange County Convention Center, de la ciudad de Orlando, Florida. Sin duda, uno de los eventos más destacados de la agenda cardiológica, contó con la participación de más de 13.000 profesionales, con destacados invitados internacionales y múltiples actividades científicas. Uno de los puntos más esperados del evento fue la presentación de los ensayos clínicos; entre los 2.719 resúmenes aceptados, fueron 20 los trabajos seleccionados para ser presentados en las sesiones de Late Breaking Clinical Trials y 17 en las sesiones de Investigación Clínica ${ }^{(1-3)}$.

Realizaremos un breve resumen de algunos de los principales trabajos científicos presentados.

- The ODYSSEY Outcomes Trial. Alirocumab in Patients After Acute Coronary Syndrome.

- Vest Prevention of Early Sudden Death Trial (VEST).

- TREAT Trial. Ticagrelor vs. Clopidogrel After Fibrinolytic Therapy in Patients With ST-Elevation Myocardial Infarction.

- NOTION. Five-Year Outcomes From the All-Comers Nordic Aortic Valve Intervention Randomized Clinical Trial in Patients with Severe Aortic Valve Stenosis.

- Clinical Outcomes of His Bundle Pacing Compared to Right Ventricular Pacing.

Palabras clave: SÍNDROME CORONARIO AGUDO

PREVENCIÓN TEMPRANA DE MUERTE SÚBITA

ESTENOSIS SEVERA DE LA VÁLVULA AÓRTICA

MARCAPASOS

\section{The best of the 2018 ACC Congress}

\section{Summary}

From March 10 to 12 of this year, the 67th annual meeting of the American College of Cardiology (ACC) was held at the Orange County Convention Center in Orlando, Florida. Undoubtedly, one of the most outstanding events of the cardiological agenda, was attended by more than 13.000 professionals, with prominent international guests and multiple scientific activities. One of the most awaited points of the event was the presentation of the clinical trials; among the 2.719 accepted abstracts, there were 20 works selected to be presented in the Late Breaking Clinical Trials sessions and 17 in the Clinical Research sessions ${ }^{(1-3)}$.

We will make a brief summary of some of the main scientific trials presented:

- The ODYSSEY Outcomes Trial. Alirocumab in Patients After Acute Coronary Syndrome.

- Vest Prevention of Early Sudden Death Trial (VEST).

- TREAT Trial. Ticagrelor vs. Clopidogrel After Fibrinolytic Therapy in Patients With ST-Elevation Myocardial Infarction.

- NOTION: Five-Year Outcomes From the All-Comers Nordic Aortic Valve Intervention Randomized Clinical Trial in Patients with Severe Aortic Valve Stenosis.

- Clinical Outcomes of His Bundle Pacing Compared to Right Ventricular Pacing.

Key words: $\quad$ ACUTE CORONARY SYNDROME

EARLY PREVENTION OF SUDDEN DEATH

SEVERE AORTIC VALVE STENOSIS

PACEMAKER

1. Editora asociada de la Revista Uruguaya de Cardiología.

2. Centro Cardiovascular Casa de Galicia. Montevideo, Uruguay.

3. Editora adjunta de la Revista Uruguaya de Cardiología.

Recibido Jun 11, 2018; aceptado Jun 28, 2018 


\section{The ODYSSEY Outcomes Trial. Alirocumab in Patients After Acute Coronary Syndrome}

El ODYSSEY Outcomes Trial fue presentado el pasado 10 de marzo, en la primera sesión de Late Breaking Clinical Trials, por el Dr. Philippe Gabriel Steg (Hospital Bichat-Claude Bernard, París, Fran-

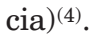

En este ensayo clínico se planteó como objetivo evaluar la seguridad y eficacia de alirocumab versus placebo en pacientes con antecedente de síndrome coronario agudo (SCA) que ya recibían tratamiento intensivo con estatinas. Después del FOURIER ${ }^{(5)}$, este es el segundo estudio randomizado que demostró una reducción significativa de eventos cardiovasculares ${ }^{(6)}$.

El alirocumab es un anticuerpo monoclonal humano que actúa inhibiendo la PCSK9. Como consecuencia, el hígado reduce la concentración de colesterol LDL (LDLc) en la sangre. Investigaciones previas han demostrado que los inhibidores de la PCSK9 reducen los niveles de LDLc a la mitad, pero el ODYSSEY Outcomes, según los investigadores, es el primer estudio en el que un inhibidor de la PCSK9 muestra un beneficio de mortalidad asociada $^{(7)}$.

Este ensayo multicéntrico, aleatorizado, doble ciego, incluyó 18.924 pacientes, provenientes de 1.315 centros (57 países), que tuvieron un SCA en los últimos 12 meses y presentaban cifras de LDLc $\geq$ $70 \mathrm{mg} / \mathrm{dl}$, no HDL colesterol $\geq 100 \mathrm{mg} / \mathrm{dl}$ o apolipoproteína $\mathrm{B} \geq 80 \mathrm{mg} / \mathrm{dl}$, después de dos a dieciséis semanas de tratamiento con estatinas a dosis altas (atorvastatina 40 a $80 \mathrm{mg}$ o rosuvastatina 20 a 40 mg) o las máximas toleradas ${ }^{(4,6,8)}$.

La mediana de edad fue de 58 años y $25 \%$ fueron mujeres. Se randomizaron en dos grupos. Un grupo recibió alirocumab $75 \mathrm{mg}$ subcutáneo cada dos semanas $(n=9.462)$ versus un grupo control que recibió placebo $(n=9.462)$. El nivel objetivo de LDLc fue 25 a 50 mg/dl. Para maximizar el número de pacientes en rango objetivo se realizó una titulación ciega de alirocumab, este se aumentó a $150 \mathrm{mg}$ cada dos semanas en pacientes con LDLc $\geq 50 \mathrm{mg} / \mathrm{dl}$ y se cambiaron a placebo aquellos con niveles de LDLc por debajo de $15 \mathrm{mg} /$ dl. Las cifras de LDLc fueron ciegas para el investigador ${ }^{(4,6,8)}$.

Después de una mediana de seguimiento de 2,8 años, los niveles de LDLc fueron de $53,3 \mathrm{mg} / \mathrm{dl}$ en el grupo alirocumab en comparación con 101,4 mg/dl en el grupo placebo, para una reducción absoluta de $54,7 \%$. El punto final primario fue un compuesto de los principales eventos cardiovasculares adversos (MACE): muerte por enfermedad coronaria, infarto agudo de miocardio (IAM) no fatal, angina inestable que requirió hospitalización o accidente cerebrovascular (ACV) isquémico; este fue significativamente menor en el grupo alirocumab versus el grupo placebo, $9,5 \%$ versus $11,1 \%$, (HR 0,85; IC95\%: $0,78-0,93 ; p=0,0003)^{(4,6,8)}$.

$\mathrm{Al}$ analizar los componentes de manera individual (punto final secundario), los investigadores encontraron una reducción significativa en la mortalidad por todas las causas, que fue de 3,5\% en el grupo de alirocumab frente a $4,1 \%$ en el grupo control (HR 0,85; IC 95\%: 0,73-0,98; p=0,026). En el grupo tratado se objetivó una tendencia no significativa a la reducción de muerte por enfermedad coronaria $(2,2 \%$ versus $2,3 \%)$ y de muerte por causa cardiovascular $(2,5 \%$ versus $2,9 \%)$. El IAM no fatal se redujo en $14 \%(\mathrm{p}=0,006)$, el ACV isquémico en $27 \%(\mathrm{p}=0,01)$ y la angina inestable en $39 \%(p=0,02)$ con alirocumab en comparación con placebo ${ }^{(4,6,8)}$.

En términos de seguridad, los eventos adversos del tratamiento se observaron en $75,8 \%$ de los tratados con alirocumab y en 77,1\% del grupo placebo. No hubo diferencias significativas en la ocurrencia de eventos adversos en la comparación de las dos ramas, salvo un aumento significativo de las reacciones locales en el punto de inyección de alirocumab de $3,8 \%$ versus $2,1 \%$ (HR 1,82 ; IC $95 \%: 1,54-2,17)^{(4,6,8)}$.

Los autores concluyen que en pacientes con SCA reciente la disminución del LDLc a niveles muy bajos con alirocumab reduce significativamente los MACE, el IAM y el ACV isquémico; también se asocia con una menor tasa de mortalidad por cualquier causa y resulta seguro y bien tolerado ${ }^{(4)}$.

En el análisis post hoc se evaluó a los pacientes según el nivel de LDLc al inicio del estudio, y los más beneficiados fueron aquellos que tenían un LDLc $\geq 100 \mathrm{mg} / \mathrm{dl}$ y recibieron tratamiento. Este subgrupo de pacientes tuvo una reducción de $24 \%$ en los MACE (reducción de 3,4\% del riesgo absoluto) y de $29 \%$ en la mortalidad por todas las causas (reducción de 1,7\% del riesgo absoluto) ${ }^{(8)}$.

"Estuvimos muy contentos de ver que el tratamiento fue efectivo y se asoció con una reducción en la mortalidad. Es notable que una intervención tan potente también sea tan segura”, dijo el Dr. P. G. Steg. "Debido a que el efecto del tratamiento fue mucho más marcado en los pacientes con el LDLc más alto, creemos que estos pacientes son los candidatos óptimos para esta terapia”(8), agregó.

Para concluir, el investigador principal refirió: "Ahora que tenemos dos ensayos que mues- 
tran consistentemente los beneficios de los inhibidores de PCSK9, y dado el beneficio de mortalidad que estamos informando aquí por primera vez, creo que estos resultados pueden cambiar la ecuación de estos medicamentos. No solo estamos ha- blando sobre la prevención de eventos no fatales, sino que realmente preservamos la vida"(8).

\section{Vest Prevention of Early Sudden Death Trial (VEST)}

La muerte súbita (MS) posinfarto agudo de miocardio (IAM) es una de las principales causas de muerte en los primeros tres meses (con mayor incidencia en el primer mes) tras el evento en pacientes con fracción de eyección del ventrículo izquierdo (FEVI) reduci$\mathrm{da}^{(9,10)}$. Sin embargo, el implante de un cardiodesfibrilador (CDI) en prevención primaria durante los primeros 40 días pos IAM no ha demostrado ser beneficioso $^{(11,12)}$, reservándose dentro de este período para casos seleccionados ${ }^{(13)}$. Las guías actuales recomiendan el CDI en prevención primaria en pacientes con FEVI $\leq 35 \%$ y clase funcional II-III, y en aquellos con FEVI $\leq 30 \%$ y clase funcional I, a pesar del tratamiento médico óptimo, 40 días pos IAM o 90 días posrevascularización y expectativa de vida mayor a un año ${ }^{(13)}$.

El chaleco cardiodesfibrilador (CCD) es un dispositivo que se utiliza bajo la ropa que monitorea continuamente el ritmo cardíaco, generando un choque eléctrico al detectar una arritmia ventricular. Su uso es indicación clase IIb en pacientes pos IAM durante el período referido, aunque la evidencia es escasa(13). Este estudio fue presentado por el Dr. Olgin, primer autor del trabajo. Es el único ensayo clínico randomizado que evalúa la eficacia del CCD en la reducción de MS en pacientes pos IAM y FEVI $\leq 35 \%$ durante los primeros 90 días tras el IAM, como puente al implante de $\mathrm{CDI}^{(14)}$.

Se incluyeron 2.302 pacientes (edad promedio 61 años, 63\% hombres) reclutados desde 2009 hasta 2017, procedentes de más de 100 centros de Estados Unidos y Europa. Los pacientes incluidos fueron dados de alta tras sufrir un IAM con FEVI $\leq 35 \%$, evaluada tras ocho horas del evento o de la angioplastia, o luego de 48 horas de realizada la cirugía cardíaca. Se randomizaron $2: 1$ a recibir CCD (LifeVest) más tratamiento médico óptimo $(\mathrm{n}=1.524)$ frente a tratamiento médico óptimo solo $(n=778)$. El objetivo del estudio fue comparar la eficacia del CCD en reducir la MS a 90 días en pacientes pos IAM con FEVI disminuida(15). El punto final primario fue la ocurrencia de MS más muerte por arritmia ventricular a los tres meses y los secundarios: mortalidad total, muerte no súbita y rehospitalizaciones por todas las causas ${ }^{(16)}$. El análisis fue realizado bajo la intención de tra$\operatorname{tar}^{(17)}$.
El promedio de seguimiento fue de 84,3 días y las poblaciones fueron similares en ambos gru$\operatorname{pos}^{(18)}$. Quienes recibieron el CCD fueron estimulados a utilizarlo el mayor tiempo posible, quitándoselo solo para ducharse; sin embargo, el promedio de uso fue de 14,1 horas diarias ${ }^{(15)}$. Al comienzo del estudio, aproximadamente $80 \%$ usaron el CCD, disminuyendo a $45 \%$ al final del seguimiento ${ }^{(14)}$.

El punto final primario fue $1,6 \%$ en el grupo randomizado a utilizar el CCD y $2,4 \%$ en el grupo control $(\mathrm{p}=0,18)$, mientras que la mortalidad total fue $3,2 \%$ y 4,9\%, respectivamente, con una reducción relativa del riesgo de mortalidad de $35,5 \%$ $(\mathrm{p}=0,04)^{(15)}$. Con respecto a los ACV fatales, su incidencia fue de $0 \%$ en el grupo con el CCD y de $0,5 \%$ en el grupo control $(p=0,01)^{(15)}$.

Otros datos a mencionar son los relacionados con la tasa de choques. Veinte pacientes tuvieron choques apropiados durante el seguimiento $(1,4 \%)$, 13 pacientes un choque y siete pacientes al menos dos. La incidencia de choques inapropiados fue baja $(0,6 \%)^{(18)}$. De estos, ocho tuvieron una descarga y dos pacientes tuvieron al menos dos ${ }^{(15)}$.

No hubo diferencias significativas en cuanto a síntomas como fatiga, dificultad para dormir, cefaleas o náuseas, aunque en el grupo randomizado a utilizar el dispositivo la incidencia de prurito, rash cutáneo y disnea fue significativamente mayor ${ }^{(15)}$.

Si bien no hay una causa clara que explique la diferencia significativa en la mortalidad total y la tasa de ACV fatales, la Dra. Sana Al-Khatib, presente en el panel de expertos durante la exposición, propuso que tal vez los pacientes que recibieron choques inapropiados o abortaron el mismo presionando el botón del CCD (70 choques abortados, 4,6\%), acudieron a la consulta médica y se les detectó fibrilación auricular (FA), iniciándose anticoagulación de forma apropiada ${ }^{(15)}$.

También en un intento por explicar la diferencia significativa en la mortalidad por todas las causas, el Dr. Olgin planteó que dada la dificultad en clasificar la MS, algunas pudieron ser catalogadas erróneamente como muertes por otras causas. "A menudo hay pocos registros y pocas autopsias. Esto puede reducir el poder para detectar las MS, pero no para la mortalidad total”, mencionó durante la presen- 
tación ${ }^{(7)}$. En entrevista con el Dr. Peter Block para ACC.org, Olgin recalcó además que el no uso del CCD se estimó en $10 \%$, aunque finalmente fue de $20 \%$, y principalmente la tasa de muertes indeterminadas fue de $5 \%$, no incluyéndose como MS pero sí en el conteo de la mortalidad total, todo lo cual disminuye el poder para detectar diferencias en la tasa de MS entre los grupos. También menciona que el CCD no solo detecta arritmias ventriculares, sino también FA, bradicardia y asistolia, permitiendo un contacto médico precoz ${ }^{(14)}$.

Pese a que el estudio no mostró diferencias en el punto final primario, el Dr. Olgin puntualizó: "Debido a la disminución de la mortalidad total, es razonable prescribir el CCD en pacientes pos IAM con FEVI reducida hasta ser reevaluados para el implante de un CDI a los 40 o 90 días". También reconoció que el beneficio sobre la mortalidad por todas las causas "debe ser interpretado con cautela", pero que "incluso con dicha cautela, la probabilidad de que la diferencia sea espuria es baja". En respuesta a la osada conclusión de Olgin, el Dr. O'Gara, panelista, señaló que más que razonable sería apropiado decir que "la utilidad (del CCD) es incierta"(15).

El Dr. Mittal, en entrevista con theheart.org| MedscapeCardiology, apuntó al bajo número de pacientes que recibieron choques apropiados, y que "un gran número de pacientes no obtuvo beneficio del mismo pero experimentó choques inapropiados o gran incomodidad"(15).

Dado que el análisis fue por intención de tratar, y debido a la baja proporción de MS, sería interesante un análisis más minucioso de los resultados, como por ejemplo, conocer si dentro de los fallecidos por MS en el grupo con el CCD hubo pacientes que no lo utilizaron, si la arritmia fatal fue tratada apropiadamente o no por el dispositivo, y la proporción de pacientes que mejoraron la función cardíaca a lo largo del seguimiento. Esperamos con entusiasmo la publicación del estudio.

\section{TREAT Trial. Ticagrelor vs Clopidogrel After Fibrinolytic Therapy in Patients With ST-Elevation Myocardial Infarction}

El estudio TREAT comparó la seguridad a corto plazo de ticagrelor frente a clopidogrel en pacientes menores de 75 años que presentaron infarto agudo de miocardio con elevación del segmento ST (IAMcEST) tratados con fibrinolíticos. El objetivo primario fue valorar el sangrado mayor, según los criterios TIMI, en los primeros 30 días $^{(19,20)}$.

Se incluyeron 3.799 pacientes provenientes de 152 centros en 10 países. Los mismos fueron randomizados a recibir ticagrelor (dosis carga de $180 \mathrm{mg}$, seguida de 90 mg cada 12 horas) o clopidogrel (300 a $600 \mathrm{mg}$, seguida de $75 \mathrm{mg}$ diarios) en las primeras 24 horas del evento coronario ${ }^{(19)}$.

Con una media de edad de 58 años, el sangrado mayor ocurrió en 14 de 1.913 pacientes $(0,73 \%)$ bajo tratamiento con ticagrelor y en 13 de 1.886 pacientes $(0,69 \%)$ bajo tratamiento con clopidogrel (diferencia absoluta 0,04\%; IC95\%: 0,49-0,58; p<0,001)(19), lo que demuestra la no inferioridad.

La tasa de sangrado fatal $(0,16 \%$ versus $0,11 \%$; $\mathrm{p}=0,67)$ e intracraneano $(0,42 \%$ versus $0,37 \%$; $\mathrm{p}=0,82$ ) fue similar en el grupo de ticagrelor y clopidogrel, respectivamente. El sangrado menor fue más frecuente con ticagrelor.
El compuesto de muerte por causas vasculares, IAM o ACV ocurrió en 76 pacientes $(4,0 \%)$ tratados con ticagrelor y en 82 pacientes $(4,3 \%)$ en el grupo de clopidogrel (HR 0,91; IC95\%: 0,67-1,25; $\mathrm{p}=0,57)^{(19)}$.

Los investigadores concluyeron que en pacientes menores de 75 años que presentaron IAMcEST, la administración de ticagrelor luego de la fibrinólisis fue no inferior a clopidogrel para sangrado mayor TIMI a los 30 días. Sin embargo, los sangrados menores fueron más frecuentes con ticagrelor, y no hubo beneficio en los resultados de eficacia(19).

Este estudio es de gran importancia sobre todo para países latinoamericanos en donde los fibrinolíticos aún son el tratamiento disponible del IAMcEST para muchos pacientes ${ }^{(21)}$.

El doctor Berwanger, autor principal del estudio, comentó lo siguiente: "Creo que los médicos, algunos de los cuales ya están usando ticagrelor, encontrarán que los resultados son tranquilizadores porque sugieren que pueden usarlo en esta población sin causar más hemorragias graves o hemorragias fatales que el clopidogrel". En relación con la eficacia, refirió: "Tendremos que esperar hasta el próximo año para poder evaluarla"(22). 


\section{NOTION: Five-Year Outcomes From the All-Comers Nordic Aortic Valve} Intervention Randomized Clinical Trial in Patients with Severe Aortic Valve Stenosis

El ensayo clínico NOTION compara los resultados del tratamiento de la estenosis aórtica severa en pacientes con bajo riesgo operatorio, utilizando el abordaje quirúrgico (SVAo), frente al implante percutáneo con la primera generación de bioprótesis autoexpandible Corevalve (TAVI)(23).

El punto final primario fue evaluar el compuesto de muerte por cualquier causa, ACV e IAM, cinco años después de la intervención ${ }^{(23)}$.

Se incluyeron 139 pacientes que recibieron TAVI y 135 que fueron a SVAo, mayores de 70 años ${ }^{(22)}$, procedentes de tres centros de Dinamarca y Suecia, con un seguimiento de $100 \%$ a cinco años. Presentaban una media de edad de 79 años, STS score medio 3\%, Log EuroSCORE 8,4\%, siendo comparables ambos grupos ${ }^{(23)}$.

A los cinco años de seguimiento no se observaron diferencias significativas en el punto final primario (39,2\% para TAVI versus $35,8 \%$ para los que recibieron SVAo, $\mathrm{p}=0,78)^{(22,23)}$. Tampoco se encuentran diferencias cuando se analiza por separado, la tasa de muerte por todas las causas $(27,7 \%$ para ambos grupos); el ACV (10,5\% de los pacientes con TAVI y $8,2 \%$ con SVAo, $p=0,67)$ y el IAM $(8,7 \%$ TAVI y $8,6 \%$ SVAo) $)^{(24)}$.

Es de destacar la elevada tasa de FA en el grupo de SVAo $(62,2$ frente a $25,2 \%$; p < 0,001) y la tasa de marcapasos en el grupo de TAVI (41,8 frente a $8,4 \% ; \mathrm{p}<0,001)^{(23)}$.

El uso de TAVI se asoció con mayores tasas de insuficiencia aórtica (Iao); 52,9\% de los pacientes presentaron insuficiencia de grado leve y $8,2 \%$ de grado moderado, en comparación con $77,4 \%$ de los pacientes con SVAo que no asociaron insuficiencia $^{(24)}$. Sin embargo, no se observó compromiso en la clase funcional de los pacientes a lo largo del seguimiento.

Se asoció con aumento de la mortalidad en pacientes con TAVI: nuevo implante de marcapaso permanente, IAo moderada a severa, edad $\geq 75$ años, sexo masculino, índice de masa corporal $\leq 30$, y STS $\geq 4 \%{ }^{(24)}$.

El ensayo NOTION es el primero en mostrar los resultados a cinco años del uso de TAVI versus SVAo en pacientes de bajo riesgo (STS $\leq 4 \%$ ). De acuerdo a los investigadores, es necesario el seguimiento a largo plazo para valorar la duración de TAVI luego de su implante(24).

Los resultados fueron presentados por el doctor Thyregod, autor principal del estudio, quien dijo lo siguiente: "Estos resultados de seguimiento a cinco años se suman al conjunto de evidencias a largo plazo que respaldan la evidencia a favor del uso de TAVI"(25).

\section{Clinical Outcomes of His Bundle Pacing Compared to Right Ventricular Pacing}

La estimulación crónica apical del ventrículo derecho (VD) genera efectos deletéreos en la estructura y función cardíacas, ya que provoca disincronía mecánica y eléctrica, asociándose a tasas más elevadas de FA, insuficiencia cardíaca (IC) y $\operatorname{mortalidad}^{(26)}$. Un subestudio del MOST (Mode Selection Trial) demostró que la estimulación ventricular derecha (EVD) mayor a $40 \%$ del tiempo se asocia a mayor incidencia de FA y riesgo de hospitalizaciones por IC, en pacientes con disfunción del nodo sinusal y QRS $<120$ $\mathrm{ms}^{(27)}$. Además, en el estudio DAVID (Dual Chamber and VVI Implantable Defibrillator) el combinado de muerte y hospitalización por IC fue menor en pacientes con estimulación en modo VVI-40 cpm de respaldo versus aquellos con programación DDDR-70 cpm, particularmente en el subgrupo con EVD mayor a $40 \%$ del tiempo ${ }^{(28)}$. A su vez, estudios recientes han referido como umbral de marcapaseo asociado a cardiomiopatía una carga de estimulación ventricular de $20 \%(29)$
Estos hallazgos han llevado a la búsqueda de otras localizaciones alternativas de estimulación ventricular, como la estimulación del haz de His (EHH), descrita por primera vez en el año $2000^{(30)}$. Con el advenimiento de electrodos y vainas de liberación más apropiadas para tal fin, sumado a publicaciones que muestran la seguridad y reproducibilidad del procedimiento, la EHH ha cobrado mayor aceptación en los últimos años ${ }^{(31)}$.

El Dr. Vijayaraman y colaboradores diseñaron este estudio observacional de cohorte con el objetivo de evaluar los resultados clínicos de la $\mathrm{EHH}$ en comparación con la EVD. Se incluyeron todos los pacientes $(n=765)$ que requirieron el implante de un marcapasos definitivo por primera vez debido a disfunción del nodo sinusal (35\%) o enfermedad de conducción auriculoventricular (AV) (65\%), en el período octubre de 2013 a diciembre de 2016. Los implantes fueron realizados en dos centros médicos pertenecientes a la misma institución, pero ubica- 
dos en distintas ciudades de Pensilvania: en Danville se realizó el implante convencional, mientras que en Wilkes-Barre el implante a nivel del His. Se excluyeron pacientes con indicación de terapia de resincronización cardíaca (TRC) o portadores de dispositivos electrónicos cardíacos ${ }^{(31)}$.

El electrodo de EHH (de fijación activa) se implantó en el VD cercano al anillo tricuspídeo a través de la vena cefálica, axilar o subclavia. Para el procedimiento, se identificó el electrograma del His con mapeo a nivel del septum AV (en caso de no detectarse se realiza pace-mapping para identificar el sitio correcto) y se implantó el electrodo en dicha posición. En caso de no lograrse una aceptable captura del His tras cuatro intentos, o si el tiempo de fluoroscopía excediera los 20 minutos, el electrodo se ubicaba en una posición no apical del VD(31).

Se analizaron 765 pacientes mayores de 18 años (edad promedio 76 años), 56\% eran hombres, 29\% refería antecedentes de IC y $50 \%$ tenía FA. La FEVI promedio inicial fue de 54,5\%. Para el análisis se dividieron los pacientes en dos grupos, uno con $\mathrm{EHH}$ (332 pacientes) y el grupo control con EVD (433 pacientes) ${ }^{(31)}$.

Las poblaciones eran similares a excepción del sexo (60\% hombres versus $52 \%$ mujeres, $\mathrm{p}<0,05)$, la presencia de FA ( $57 \%$ versus $45 \%$, p < 0,05 ), y la duración del QRS basal (104,5 ms versus $110 \mathrm{~ms}$, $\mathrm{p}<0,01$ ) en el grupo con EHH y en el grupo control, respectivamente. En el grupo de EHH se realizó un seguimiento promedio de 754 días, y en el grupo control de 648 días. El marcapaseo permanente en el grupo con EHH se logró en el 91,6\% de los pacientes (304 de 332), mientras que en el grupo de EVD la estimulación apical se efectuó en 40,6\% y la no apical en $59,4 \%{ }^{(31)}$.

El objetivo primario fue el combinado de mortalidad, primer episodio de internación por IC y el upgrading a TRC, que se evidenció en $25 \%$ del grupo EHH y 31,6\% del grupo EVD (HR 0,71, p=0,02)(31).

En el análisis de subgrupos según la carga de estimulación ventricular, se observó que en aquellos con carga de estimulación $>20 \%$ del tiempo, el punto final primario se objetivó en $25,3 \%$ del grupo de EHH y $35,6 \%$ del control (HR 0,65, p=0,02), mientras que en aquellos con estimulación $\leq 20 \%$ del tiempo, no hubo diferencias significativas entre ambos grupos ( $22 \%$ versus $23,7 \%, \mathrm{p}=0,34)^{(31)}$.

Los objetivos secundarios evaluaron la mortalidad y las internaciones por IC. Los pacientes con EHH tuvieron menores tasas de internación por IC $(12,4 \%)$ comparados con el subgrupo con EVD $(17,6 \%)(H R 0,633, p=0,02)$, debido principalmente al subgrupo con carga de estimulación ventricular $>20 \%$, sin diferencias significativas en aquellos pacientes con carga $\leq 20 \%$. Hubo una tendencia a menor mortalidad en el grupo de $\mathrm{EHH}$ (17,2\% versus $21,4 \%, p=0,06$ ). Por otro lado, en este grupo hubo mayor tiempo de procedimiento (70 min versus $55 \mathrm{~min}$, $\mathrm{p}<0,01$ ) y de fluoroscopía (10 min versus $7 \mathrm{~min}$, $\mathrm{p}<0,01)$. El upgrading a TRC se realizó en un porcentaje bajo de pacientes: un paciente $(0,3 \%)$ en el grupo de EHH y seis $(1,4 \%)$ en el grupo de EVD. El grupo de $\mathrm{EHH}$ necesitó mayor umbral de captura, tanto al implante $(1,30 \mathrm{~V}$ vs $0,59 \mathrm{~V}, \mathrm{p}<0,01)$ como al final del seguimiento $(1,56 \mathrm{~V}$ vs $0,76 \mathrm{~V}, \mathrm{p}<0,01)$. El ancho del QRS marcapaseado fue menor en el grupo con $\mathrm{EHH}$ (128 ms vs $166 \mathrm{~ms}, \mathrm{p}<0,01)^{(31)}$.

Dentro del grupo con $\mathrm{EHH}$, las razones por las cuales no se implantó el electrodo a nivel del haz de His fueron: imposibilidad de mapear correctamente el His (8 pacientes), incapacidad de fijarlo apropiadamente (3 pacientes), bloqueo AV infranodal sin posibilidad de reclutar el His-Purkinje distal (11 pacientes) y altos umbrales de captura a corregir en seis pacientes ${ }^{(31)}$.

Debido a falla de captura o umbrales inaceptablemente elevados, se requirió la revisión de los cables ventriculares en 14 pacientes con $\mathrm{EHH}(4,2 \%)$ en cuatro meses de seguimiento y en dos pacientes con EVD (0,5\%) en los primeros 30 días tras el procedimiento. Se reportaron tres pacientes con derrame pericárdico en el grupo EVD que requirieron pericardiocentesis y ninguno en el grupo EHH. Ambos grupos presentaron un caso de infección con necesidad de remoción del dispositivo/cable. El agotamiento prematuro del generador ocurrió en un paciente del grupo EHH a 3,5 años tras el implan$\mathrm{te}^{(31)}$.

En entrevista con MedPageToday, Vijayaraman concluyó: "La estimulación del haz de His es una forma de marcapaseo más fisiológica, posible y segura. Reduce la incidencia de IC e incluso podría reducir la mortalidad comparada con la estimulación del ventrículo derecho. Este es el estudio más grande publicado sobre EHH con resultados clínicos a largo plazo"(32).

Tras la presentación del trabajo, uno de los panelistas, el Dr. Kristen Patton, mencionó: "No exagero si digo lo excitados que estamos en la electrofisiología en relación con la $\mathrm{EHH}$, y qué maravillosa y elegante solución es al problema de la disincronía inducida por el marcapaseo"(33).

Por otro lado, el Dr. Abdelrahman, presentador del trabajo, oportunamente señaló: "Creemos que la EHH es mejor que la EVD, pero aún se requieren estudios randomizados para confirmar nuestra hipótesis"(33). 


\section{Bibliografía}

1. ACC.18 67th Scientific Session \& Expo [Internet]. Washington: American College of Cardiology Foundation; 2018 [Consulta: 29 Junio 2018]. Disponible en: https://accscientificsession.acc.org.

2. ACC 2018 Late-Breaking Trials [Internet]. Illinois: Diagnostic and Interventional Cardiology; 2018 [Consulta: 29 Junio 2018]. Disponible en: https://www. dicardiology.com/content/acc-2018-late-breakingtrials.

3. What to expect ACC 2018 [Internet].Cardiovascular Business; 2018 [Consulta: 29 Junio 2018]. Disponible en: https:/www.cardiovascularbusiness.com/ topics/acute-coronary-syndrome/what-expect-acc2018.

4. Schwartz GG, Szarek M, Bhatt DL, Bittner V, Díaz R, Edelberg J, et al. The ODISSEY Outcomes trial: topline results Alirocumab in patients after acute coronary syndrome [Internet]. Washignton: American College of Cardiology; 2018 [Consulta: 2 Julio 2018]. Disponible en: http://www.acc.org/ / media/Clinical/PDF-Files/ApprovedPDFs/2018/03/05/ACC18_Slides/March10 Sat/9amET-ODYSSEY-Outcomes-acc-2018.pdf.

5. Sabatine MS, Giugliano RP, Keech AC, Honarpour N, Wiviott SD, Murphy SA, et al. Evolocumab and clinical outcomes in patients with cardiovascular disease. N Engl J Med 2017; 376(18): 1713-22. doi: 10.1056/NEJMoa1615664

6. Dalmau González-Gallarza R. Lo mejor de ACC.18: ODISSEY Outcomes. 2018 Mar 10 [Consulta: 2 Julio 2018]. En: Sociedad Española de Cardiología: Cardiología hoy blog [Internet]. Madrid: SEC. 2018 - [Aprox.1 pantalla] . Disponible en: https://secardiologia.es/multimedia/blog/9342-lo-mejor-de-acc-18-odyssey-outcomes.

7. Diagnostic and Interventional Cardiology;2018. Alirocumab in patients after acute coronary syndrome [Internet]. Illinois: [Consulta: 2 Julio 2018]. Disponible en: https://www.dicardiology.com/ content/alirocumab-reduces-cardiovascular-eventsafter-acute-coronary-syndrome.

8. American College of Cardiology. ODISSEY Outcomes: resuls suggest use of PCSK9 inhibitor reduces CV events, LDL-C in ACS patiens [Internet].Washington: ACC; 2018 [Consulta: 3 Junio 2018]. Disponible en: http://www.acc.org/latest-in-cardiology/ articles/2018/03/05/15/53/sat-9am-odyssey-outcomescv-outcomes-with-alirocumab-after-acs-acc-2018.

9. Adabag AS, Therneau TM, Gersh BJ, Weston SA, Roger VL. Sudden death after myocardial infarction. JAMA 2008;300 (17): 2022-9. doi: 10.1001/ jama.2008.553

10. Solomon SD, Zelenkofske S, McMurray JJ, Finn PV, Velazquez E, Ertl G, et al. Sudden death in patients with myocardial infarction and left ven- tricular dysfunction, heart failure, or both. N Engl J Med. 2005;352(25):2581-8.

11. Hohnloser SH, Kuck KH, Dorian P, Robert RS, Hamptom JR, Hatala R, et al. Prophylactic use of an implantable cardioverter-defibrillator after acute myocardial infarction. N Engl J Med. 2004;351(24): 2481-8.

12. Steinbeck G, Andresen D, Seidl K, Karlheinz S, Brachmann J, Hoffmann E, et al. Defibrillator implantation early after myocardial infarction. $\mathrm{N}$ Engl J Med. 2009;361 (15):1427-36. doi: 10.1056/NEJMoa0901889.

13. Al-Khatib SM, Stevenson WG, Ackerman MJ, Bryant WJ, Callans DJ, Curtis AB, et al. 2017 AHA/ACC/HRS Guideline for management of patients with ventricular arrhythmias and the prevention of sudden cardiac death: a report of the American College of Cardiology/American Heart Association Task Force on Clinical Practice Guidelines and the Heart Rhythm Society. J Am Coll Cardiol 2017. pii: S0735-1097(17)41306-4. doi: 10.1016/j.jacc.2017.10.054

14. American College of Cardiology. VEST: Wearable Cardioverter-Defibrillator Reduces Total Mortality, Not Sudden Deaths Post MI [Internet]. Washington: ACC; 2018 [Consulta: 21 Mayo 2018]. Disponible en: http://www.acc.org/latest-in-cardiology/ articles/2018/03/05/15/53/sat-9am-vest-efficacyof-a-wearable-cardioverter-defibrillator-aftermi-acc-2018.

15. Medscape. Stiles S. VEST: No sudden death benefit early Post-MI from wearable defibrillator [Internet]. New York: Medscape; 2018 [Consulta: 21 Mayo 2018]. Disponible en: https://www.medscape.com/ viewarticle/893753.

16. Kumbhani DJ. Vest Prevention of Early Sudden Death Trial - VEST [Internet]. Washignton: ACC; 2018 [Consulta: 21 Mayo 2018]. Disponible en: http://www.acc.org/latest-in-cardiology/clinicaltrials/2018/03/09/08/06/vest.

17. Olgin JE, Pletcher M, Vittinghioff E, Borrggrefe M, Buxton A, Chung E, et al. 401-10-Efficacy of a wearable cardioverter-defibrillator after myocardial infarction: results of the Vest prevention of early sudden death trial (VEST) [Abstract]. Orlando: ACC; 2018 [Consulta: 21 Mayo 2018]. Disponible en: http://www.abstractsonline.com/pp8/\#!/4496/presentation/41742.

18. Rao SV, Olgin JE, Lee BK, Curti AB. Preventing SCD With a WCD: Reviewing the Results of the VEST Trial [Internet]. New York: Medcape; 2018 [Consulta: 21 Mayo 2018]. Disponible en: https: // www.medscape.org/viewarticle/894113.

19. Berwanger O, Nicolau JC, Carvalho AC, Jiang L, Goodman SG, Nicholls SJ, et al. Ticagrelor vs Clopidogrel after fibrinolytic therapy in patients 
with ST-elevation myocardial infarction. A randomized clinical trial. JAMA Cardiol. 2018;3(5): 391-9. doi:10.1001/jamacardio.2018.0612.

20. Alegría Barrero E. Lo mejor de ACC.18: TREAT 2018 Marzo 11 [Consulta: 21 Mayo 2018]. En: Sociedad Española de Cardiología. Cardiología hoy blog. Madrid: SEC; 2018 [Aprox.1 p.]. Disponible en: https://secardiologia.es/multimedia/blog/ 9345-lo-mejor-de-acc-18-treat.

21. Bahit C. Estudios de Latinoamérica presentados en el Congreso del American College of Cardiology (ACC) de 2018 [Internet]. New York: Medscape; 2018 [Consulta: 3 Julio 2018]. Disponible en: https://espanol.medscape.com/verarticulo/5902459.

22. Diagnostic and Interventional Cardiology; 2018. Ticagrelor has comparable safety to Clopidogrel after heart attack [Internet]. Illinois [Consulta: 3 Julio 2018]. Disponible en: https://www.dicardiology.com/content/ticagrelor-has-comparable-safetyclopidogrel-after- heart-attack.

23. Alegría Barrero E. Lo mejor de ACC.18: NOTION 2018 Marzo 11 [Consulta: 3 Julio 2018]. En: Sociedad Española de Cardiología. Cardiología hoy blog. Madrid: SEC; 2018 [Aprox.1 p.]. Disponible en: https://secardiologia.es/multimedia/blog/9339lo-mejor-de-acc-18-notion.

24. American College of Cardiology. NOTION: Lower-risk patients undergoing TAVR, SAVR have similar outcomes after five years [Internet]. Washignton: ACC; 2018 [Consulta: 3 Julio 2018]. Disponible en: http://www.acc.org/latest-in-cardiology/articles/ 2018/03/05/15/53/sat-1215pm-notion-5-year-outcomes-of-all-comers-nordic-aortic-valve-intervention-acc-2018.

25. Diagnostic and Interventional Cardiology. CoreValve TAVR system shows strong long-term performance in clinical trials [Internet]. Illinois: Diagnostic and Interventional Cardiology; 2018 [Consulta: 3 Julio 2018]. Disponible en: https://www.dicardiology.com/content/corevalve-tavr-systemshows-strong-long-term-performance-clinical-trials.

26. Tops LF, Schalij MJ, Bax JJ. The effects of right ventricular apical pacing on ventricular function and dyssynchrony implications for therapy. J Am Coll Cardiol 2009;54(9):764-76.

doi: 10.1016/j.jacc.2009.06.006

27. Sweeney MO, Hellkamp AS, Ellenbogen KA, Greenspon AJ, Freedman AR, Lee KL, et al. Adverse effect of ventricular pacing on heart failure and atrial fibrillation among patients with normal baseline QRS duration in a clinical trial of pacemaker therapy for sinus node dysfunction. Circulation 2003;107 (23):2932-7.

28. Wilkoff BL, Cook JR, Epstein AE, Green HL, Hallstrom AP, Hsia H, et al. Dual-chamber pacing or ventricular backup pacing in patients with an implantable defibrillator: the Dual Chamber and VVI Implantable Defibrillator (DAVID) trial. JAMA 2002; 288 (24):3115-23.

29. Kiehl EL, Makki T, Kumar R, Gumber D, Kwon DH, Rickard JW, et al. Incidence and predictors of right ventricular pacing-induced cardiomyopathy in patients with complete atrioventricular block and preserved left ventricular systolic function. Heart Rhythm 2016;13(12):2272-8. doi: 10.1016/j.hrthm.2016.09.027.

30. Deshmukh P, Casavant D, Romanyshyn M, Anderson K. Permanent direct His bundle pacing: a novel approach to cardiac pacing in patients with normal His-Purkinje activation. Circulation 2000; 101(8):869-77.

31. Abdelrahman M, Subzposh FA, Beer D, Durr B, Naperkowski A, Sun H, et al. Clinical outcomes of his bundle pacing compared to right ventricular pacing. J Am Coll Cardiol. 2018;71(20):2319-30. doi: 10.1016/j.jacc.2018.02.048

32. George J. ACC: His Bundle Pacing May Best RVP for Permanent Pacemaker Disponible [Internet]. New York: Medpage Today; 2018 [Consulta 10 Mayo 2018]. Disponible en: https://www.medpagetoday.com/ mastery-of-medicine/art-and-science-of-hf/71690.

33. Stiles S. His-Bundle pacing for bradycardia may cut HF hospitalization risk compared with RV pacing [Internet]. New York: Medcape; 2018 [Consulta: 10 Mayo 2018]. Disponible en: https://www. medscape.com/viewarticle/894035. 